Documentation et bibliothèques

\title{
Les périodiques en généalogie et en histoire de la famille : des ressources très utiles, mais peu exploitées
}

\section{Denis Boudreau}

Volume 59, numéro 2, avril-juin 2013

URI : https://id.erudit.org/iderudit/1033223ar

DOI : https://doi.org/10.7202/1033223ar

Aller au sommaire du numéro

\section{Éditeur(s)}

Association pour l'avancement des sciences et des techniques de la documentation (ASTED)

\section{ISSN}

0315-2340 (imprimé)

2291-8949 (numérique)

Découvrir la revue

\section{Citer cet article}

Boudreau, D. (2013). Les périodiques en généalogie et en histoire de la famille : des ressources très utiles, mais peu exploitées. Documentation et bibliothèques, 59(2), 114-119. https://doi.org/10.7202/1033223ar
Résumé de l'article

En héritant de l'ensemble de la collection de ressources en généalogie de la salle Gagnon de la défunte Bibliothèque centrale de Montréal en 2005, le Centre d'archives de Montréal reçoit notamment une grande quantité de revues de ce domaine. Cette acquisition enrichit avantageusement la collection de revues du Centre, jusque-là plutôt modeste. Ce sont plus de 280 titres différents qui forment aujourd'hui cette collection qui compte bien sûr des publications québécoises, mais aussi canadiennes, américaines et européennes. Même s'il n'est pas rare que l'on voie des usagers se consacrer au dépouillement des numéros de l'une ou l'autre de ces revues, il serait faux de dire que le potentiel de ces périodiques pour la recherche en généalogie est bien connu. Notre objectif est donc de valoriser cette collection.
Tous droits réservés (C) Association pour l'avancement des sciences et des techniques de la documentation (ASTED), 2013
Ce document est protégé par la loi sur le droit d'auteur. L'utilisation des services d'Érudit (y compris la reproduction) est assujettie à sa politique d'utilisation que vous pouvez consulter en ligne.

https://apropos.erudit.org/fr/usagers/politique-dutilisation/ 


\title{
Les périodiques en généalogie et en histoire de la famille : des ressources très utiles, mais peu exploitées
}

\author{
DENIS BOUDREAU \\ Bibliothécaire, Centre d'archives de Montréal \\ Bibliothèque et Archives nationales du Québec \\ Denis.boudreau@banq.qc.ca
}

\section{La collection de périodiques du Centre d'archives de Montréal}

En héritant de l'ensemble de la collection de ressources en généalogie de la salle Gagnon ${ }^{*}$ de la défunte Bibliothèque centrale de Montréal en 2005, le Centre d'archives de Montréal reçoit notamment une grande quantité de revues de ce domaine. Cette acquisition enrichit avantageusement la collection de revues du Centre, jusque-là plutôt modeste. Ce sont plus de 280 titres différents qui forment aujourd'hui cette collection qui compte bien sûr des publications québécoises, mais aussi canadiennes, américaines et européennes. Même s'il n'est pas rare que l'on voie des usagers se consacrer au dépouillement des numéros de l'une ou l'autre de ces revues, il serait faux de dire que le potentiel de ces périodiques pour la recherche en généalogie est bien connu. Notre objectif est donc de valoriser cette collection.

* Ébauchée par Philéas Gagnon à la fin du xix ${ }^{e}$ siècle, la collection de documents en généalogie est cédée à la Ville de Montréal en 1910. C'est sur elle que s'appuie en partie l'établissement de la Bibliothèque centrale de Montréal inaugurée en 1917. Par la suite, on y crée une salle de recherche spécialisée en généalogie : la salle Gagnon. Pour plus d'information, voir M. Baboyanté. 1996. La Bibliothèque de la Ville de Montréal, la collection Gagnon et son fondateur Philéas Gagnon. Cahiers d'histoire du Québec au XX $x^{e}$ siècle, $\mathrm{n}^{\circ} 6$, p. 67-82.
T A RECHERCHE EN GÉNÉALOGIE EST pour plusieurs synonyme de vieux documents d'archives : aregistres paroissiaux, greffes de notaires, vieilles photos... On se réfère aussi souvent aux documents publiés tels que les monographies familiales et les dictionnaires de familles, mais on ne peut en dire autant des revues et bulletins. S'il est vrai que les journaux sont fortement valorisés dans le domaine, notamment grâce aux notices nécrologiques, ce n'est pas le cas des autres types de périodiques. Pourtant, ces documents peuvent s'avérer d'une grande richesse en information de toute sorte pour le généalogiste qui souhaite approfondir sa recherche, comme pour le bibliothécaire qui offre des services au chercheur, d'autant plus qu'il existe un grand nombre et une grande variété de ce type de publications en généalogie et en histoire de la famille.

Le présent article a pour objectif de démontrer l'utilité de cette documentation, tout en présentant les difficultés liées à son utilisation. Notre analyse se fonde essentiellement sur notre quotidien en tant que bibliothécaire dans un milieu abondamment fréquenté par les généalogistes, soit le Centre d'archives de Montréal de Bibliothèque et Archives nationales du Québec (BAnQ).

\section{Les périodiques en généalogie : une offre des plus diversifiées}

Revues spécialisées ou généralistes, bulletins ou feuillets d'information, textes de provenance locale, régionale ou nationale : l'offre des périodiques en généalogie est très diversifiée (Figure 1). Ces publications, qui émanent généralement d'une société de généalogie ou d'une association de famille, sont très nombreuses et les quelques centaines de titres différents que possède le Centre d'archives de Montréal ne représentent qu'une infime portion des périodiques consacrés à des régions ou à des familles spécifiques. Une société de généalogie, une publication: c'est presque une règle dans ce domaine. Au Québec, où on trouve près de 70 sociétés de généalogie et un peu plus de 160 associations de famille ${ }^{1}$, on dénombre plus de 200 titres (actifs ou non).

1. La Fédération québécoise des sociétés de généalogie (FQSG) et la Fédération des familles souches du Québec (FFSQ) comptaient respectivement 68 et 163 membres en 2012. 
Figure 1

Répartition des périodiques (actifs et non actifs) du Centre d'archives de Montréal (octobre 2012)

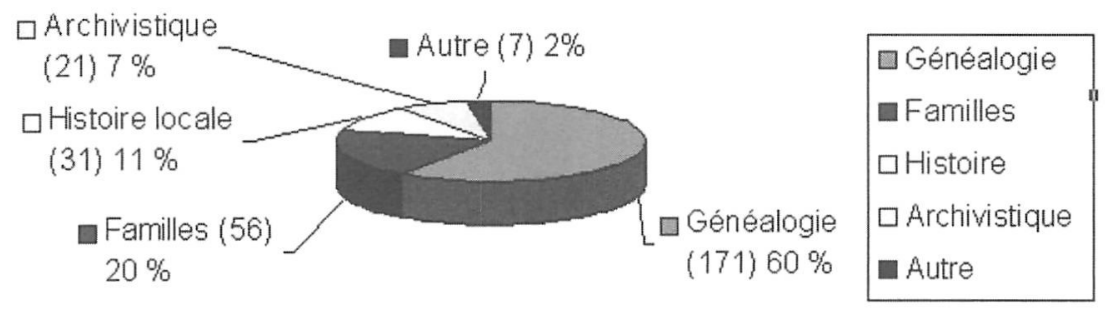

Et c'est sans compter les publications issues des autres provinces canadiennes, des états américains et des pays d'Europe.

\section{Mémoires et L'ancêtre au Québec}

Les revues Mémoires (Société généalogique canadienne-française [SGCF]) et L'ancêtre (Société de généalogie de Québec [SGQ]) comptent parmi les plus anciennes du domaine au Québec, la première étant publiée depuis 1944 et la seconde depuis 1974. La plupart des autres titres québécois apparaissent vers la fin des années 1970 et au début des années 1980. Dès la fin du XIX ${ }^{e}$ siècle, d'autres périodiques à caractère historique se sont toutefois consacrés à la généalogie à l'occasion. C'est le cas notamment du Bulletin des recherches historiques (1895-1968), de la publication Les Cahiers de Dix, fondée en 1936, puis de la Revue d'histoire de l'Amérique française, fondée en 1947.

\section{Des sociétés, des régions et des familles...}

La diversité géographique des périodiques traitant de généalogie ou d'histoire de la famille est aussi un atout non négligeable. Vous êtes à la recherche d'un ancêtre qui a migré ailleurs en Amérique? Il se peut que l'on trouve une société de généalogie dans la région concernée. À défaut de trouver un article d'une grande pertinence dans la publication de cette société, peutêtre en tirerez-vous des pistes de recherches (ressources, contacts, etc.). Un ancêtre d'une branche familiale vous cause des maux de tête? La brochure ou le bulletin de l'une des multiples associations de famille contient peut-être des réponses à vos questions. Avec plusieurs milliers de regroupements de généalogie dans le monde, la piste des périodiques n'est donc pas à négliger².

2. Dans la quatrième édition du Directory of Family Associations (Elizabeth Petty Bently et Deborah Ann Carl, 2001), on recense près de 6000 associations de famille aux États-Unis. L'édition 2012 du répertoire de Dina C. Carson, Directory of Genealogical and Historical Societies, Libraries and Periodicals in the US and

\section{Des revues d'une mer à l'autre...}

Au Canada, on trouve dans chacune des provinces un regroupement de généalogie; tous produisent un périodique. Mentionnons, par exemple, The Nova Scotia Genealogist (depuis 1983), The British Colombia Genealogist (depuis 1971), la publication Families (depuis 1971) en Ontario, ainsi que les revues Generations au Nouveau-Brunswick (depuis 1983) et au Manitoba (depuis 1976). Aux États-Unis, il n'est pas rare que ce type d'organisme produise sa propre publication. Ici, on se doit de mentionner la National Genealogical Society et sa revue NGS Magazine, mais aussi la publication American Ancestors, produite par la New England Historic Genealogical Society. Dans le domaine de la généalogie nord-américaine, cette société, avec ses 25000 membres et ses 50 employés, est certainement l'une des plus importantes. D'ailleurs, on lui doit aussi l'un des plus anciens titres en généalogie: The New England Historical and Genealogical Register (depuis 1847).

\section{En Amérique du Nord francophone, acadienne, juive, polonaise, etc.}

En Amérique française, les publications AmericanCanadian Genealogist (American-Canadian Genealogical Society, depuis 1973), Links (Vermont French-Canadian Genealogical Society, depuis 1996) et Quarterly (French Canadian / Acadian Genealogists of Wisconsin, depuis 1987) sont aussi à noter, tout comme l'éphémère French Canadian and Acadian Genealogical Review (Centre canadien des recherches généalogiques, Québec, publié de 1968 à 1981). Et il ne faudrait pas oublier la revue franco-ontarienne Le Chaînon (depuis $1983)^{3}$ et les quelques titres acadiens ${ }^{4}$. Toujours pour

Canada, Iron Gate Publishing, recense plus de 20000 sociétés de généalogie dans le monde.

3. Au printemps 2011, la Société franco-ontarienne d'histoire et de généalogie et le Regroupement des organismes du patrimoine franco-ontarien se sont réunis sous le vocable Réseau du patrimoine franco-ontarien (RPFO) <http://www.rpfo.ca/fr/ Bref-Historique_5> [consulté le 31 octobre 2012].

4. Entre autres: Les Cahiers (Société historique acadienne, depuis 1961), La Revue de la Société historique Nicolas Denys (depuis 1970) et celle de la Société historique du Madawaska (depuis 1982), ainsi que Sur l'Empremier de la Société historique de la Mer Rouge (depuis 1981). 


\section{Figure 2}

Sociétés et regroupements de généalogie - Périodiques du Centre d'archives de Montréal*. Titres actifs en date de décembre 2012.

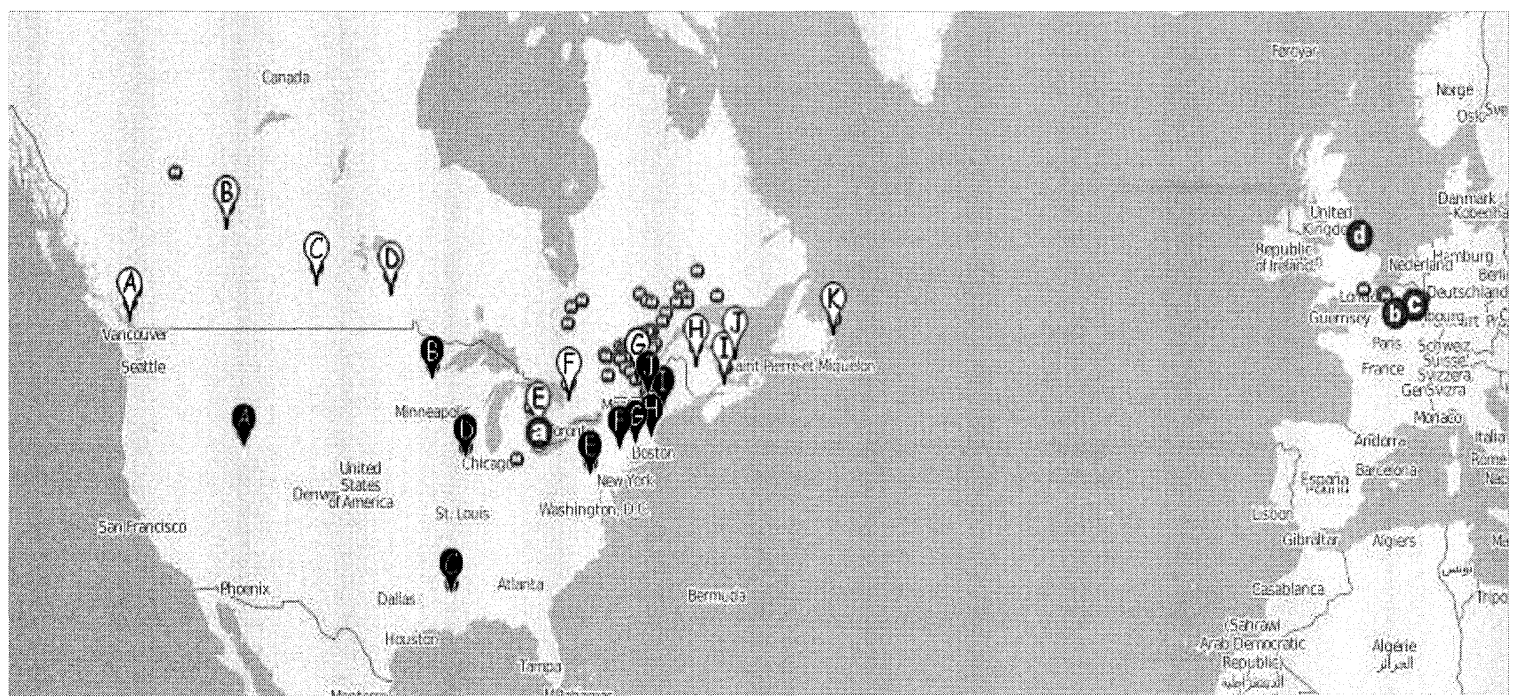

Regroupements de généalogie provinciaux canadiens: A) The British Columbia genealogist (ColombieBritannique); B) Relatively Speaking (Alberta); C) Bulletin (Saskatchewan); D) Generations (Manitoba); E) Families (Ontario); F) Le Chaînon (Franco-Ontarien); G) Info-généalogie (Québec); H) Generations (Nouveau-Brunswick); I) Nova Scotia Genealogist (Nouvelle-Écosse); J) Newsletter/P.E.I Genealogical Society (Île-du-Prince-Édouard) ; K) The Newfoundland Ancestor (Terre-Neuve-et-Labrador).

Regroupements de généalogie, États américains: A) Crossroads (Utah); B) Minnesota genealogist ; C) The Louisiana Genealogical Register; D) Illinois State Genealogical Society Quarterly; E) National Genealogical Society Quarterly; F) Connecticut Nutmegger; G) Rhode Island Roots; H) MASSOG (Massachusetts) ; I) New England Historical and Genealogical Register; J) New Hampshire Genealogical Record.

Périodiques généralistes : a) Family Chronicle ; b) Family Tree Magazine ; d) Gé-Magazine ; c) La Revue française de généalogie.

Représente un regroupement de généalogie local ou régional.

* Carte réalisée à l'aide du système de gestion de contenus sous licence libre Drupal $><$ http://www.drupal.org/ $>$, du module complémentaire GetLocations $<$ http://drupal. org/project/getlocations> et des interfaces de programmation Google Maps Javascript, version 3 <https://developers.google.com/maps/documentation/javascript/ reference?hl=fr-CA $>$ et Leaflet $\langle$ http://leafletjs.com $/\rangle$.

les groupes ethniques ou culturels, on peut mentionner le périodique anglo-québécois Connections (depuis 1978) de la Quebec Family History Society. Au-delà des publications consacrées aux racines françaises et britanniques de l'Amérique du Nord, on peut trouver des titres destinés à plusieurs autres groupes sociaux et ethniques, par exemple: Avotaynu (depuis 1985), pour la généalogie de la diaspora juive; Afro-American Historical and Genealogical Society Journal (depuis 1980 ?), pour celle des Noirs américains; Swedish American Genealogist (depuis 1981), pour la généalogie américano-suédoise; Rodziny (depuis 1977 ?), consacré aux Américano-Polonais, et Germaniques, Ahnengalerie (depuis 1981), consacré aux familles québécoises d'origine germanique.

\section{Des informations, des détails et des conseils}

Bien que le quotidien des organismes qui publient ces revues spécialisées occupe généralement une part importante du contenu (nouvelles, événements à venir, etc.), la formation et la méthodologie n'y sont pas négligées. Elles y sont traitées notamment par la présentation de cas problèmes, ainsi que d'outils et de sources particulières, ou encore sous forme de questions et réponses. On y trouve aussi des articles biographiques, des lignées généalogiques et des articles de nature historique. N’oublions pas les textes consacrés à la présentation de nouvelles ressources (documents, bases de données, etc.), aux nouvelles acquisitions des salles de recherche et aux comptes rendus de livres. Ces articles peuvent s'avérer très utiles pour la prestation de services en généa- 
logie. Certes, toutes ces publications n'ont pas la même rigueur : certaines revues ont un caractère plus professionnel, alors que l'aspect amateur est d'une grande évidence pour d'autres. Un certain nombre d'entre elles sont des publications plus généralistes et informatives. Dans l'ensemble, il s'agit surtout de textes qui présentent des cas ou des problématiques spécifiques. Tout cela relève sans doute pour l'essentiel du dynamisme de l'organisme en question.

Le domaine peut aussi compter sur quelques périodiques qui ne sont pas directement le fruit d'un regroupement de généalogie, mais plutôt d'une entreprise commerciale qui cherche à atteindre un plus grand public. Si nous pouvons citer deux exemples pour la France, la Revue française de généalogie (depuis 1979) et Généalogie Magazine (depuis 1982), au Québec et dans le reste de l'Amérique du Nord, il n'y a pas de publication de ce type en français. Sans surprise, les exemples en anglais sont plus nombreux: la revue canadienne et américaine Family Chronicle (depuis 1996), la revue britannique Family Tree Magazine (depuis 1984) et son homonyme américain (depuis 1999), ainsi que le périodique canado-américain Internet Genealogy (depuis 2006), en sont les meilleurs exemples.

\section{Des ressources méconnues et pas toujours faciles à trouver}

D'abord destinées aux membres de l'organisme qui les produit, ces publications sont généralement très peu diffusées à l'extérieur de leur territoire ou du milieu de la généalogie. Si la plupart des sociétés de généalogie participent à des échanges de numéros, les bibliothèques publiques ne semblent pas avoir pour habitude de s'abonner à ce type de publication ${ }^{5}$. De plus, ces regroupements ne respectent pas systématiquement l'obligation du dépôt légal. Il est donc possible que plusieurs titres ne soient pas présents dans les catalogues Iris de BAnQ, AMICUS de Bibliothèque et Archives Canada ou dans d'autres catalogues nationaux. Repérer une revue consacrée à une région spécifique n'est donc pas simple, d'autant plus que bon nombre de ces publications ne possèdent pas de numéro ISSN et que l'information que l'on trouve dans un répertoire tel qu'Ulrichsweb.com n'est pas toujours actualisée.

Quelques outils peuvent nous aider à combler ces lacunes. D'abord, au nombre des survivants de l'époque des répertoires papier annuels, on peut mentionner deux ressources: le répertoire de Dina C. Carson, Directory of Genealogical and Historical Societies, Libraries and Periodicals in the US and Canada (Iron Gate Publishing, 1996 ?-[2012]) et le répertoire The Family and Local History Handbook (York, Angleterre, Robert

5. Notre propos s'appuie sur une recherche faite en octobre 2012 dans le catalogue en ligne des bibliothèques de cinq municipalités québécoises : Montréal, Laval, Longueuil, Québec et Rimouski.
Blatchford Publishing, 2001-[2012]). En ligne, on se référera au répertoire de ressources généalogiques canadiennes de Bibliothèque et Archives Canada (AVITUS: $<$ http://www.collectionscanada.gc.ca/base-de-donnees/ avitus/index-f.html $>$ ) et à celui de la Bibliothèque du Congrès américaine (portail de la Local History and Genealogy Reading Room - Humanities and Social Science Division ${ }^{6}$ ). Le répertoire AVITUS peut d'autant plus être utile qu'il n'existe pas au Canada d'organisme pancanadien, similaire à la Fédération de sociétés de généalogie du Québec, regroupant les sociétés de généalogie ou les associations de famille. Dans un ultime recours, le service de référence du centre de généalogie de la bibliothèque américaine Allen County Public Library, en Indiana (<http://www.genealogycenter. org >), peut aussi être utile pour la recherche d'un périodique puisqu'on y trouve l'une des plus importantes collections de ce genre dans le monde anglo-saxon ${ }^{7}$.

\section{Une question d'index}

Trouver un périodique pertinent à une région, c'est une chose. Repérer un texte ou un article portant sur un ancêtre ou une famille, c'est une autre histoire. D'autant plus que des outils tels le Canadian Periodical Index (Gale Cengage Learning), Repère (Services documentaires multimédia) ou Eureka (Cedrom-SNi) ne sont pas très utiles pour la recherche en histoire des familles ${ }^{8}$. Pour retracer correctement ce type de documents, il est donc nécessaire de se tourner vers des outils spécialisés et moins connus dans le monde des bibliothèques publiques.

Dans la littérature du domaine, le Periodical Source Index (PERSI) est certainement la base de données la plus fréquemment citée. Avec plus de 11000 titres et 2,25 millions d'articles recensés (surtout en anglais, mais aussi en français ${ }^{9}$ ), c'est sans aucun doute la plus importante. Conçu par la Allen County Public Library en 1986, cet outil a été longtemps distribué sous forme d'imprimé (jusqu'en 1997), puis sur CD-ROM, et il est de nos jours uniquement disponible par l'entremise des bases de données commerciales HeritageQuestOnline et Ancestry.com/Ancestry.ca. Étrangement, PERSI n'est pas offert dans la version institutionnelle de cette dernière base de données, Ancestry Library Edition, ce qui n'est pas sans poser un dilemme aux institutions qui ne

6. On y trouve notamment cette rubrique : «Genealogical Periodicals in LH\&G : A Guide for Research », <http://www.loc.gov/rr/genealogy/bib_guid/genperiogene. html $>$ [consulté le 20 août 2012].

7. On parle dans ce cas de plus de 3200 abonnements courants et de plus de 4100 titres différents relatifs à la généalogie et à l'histoire des familles (Source: J. Swan. 2004. The Librarian's Guide to Genealogical Services and Research. New York, Neal-Schuman. p. 46.

8. Les publications de ce domaine sont peu présentes dans ces bases de données. La revue Mémoires de la SGCF est l'exception qui confirme la règle, dans le cas de Repère.

9. De ces 11000 titres, environ 3000 ne sont plus actifs. Source: Réponse à un courriel soumis au service de référence de la bibliothèque ("Ask a Genealogy Librarian, "<genealogy@acpl.lib.in.us>), le 21 août 2012. 
souhaitent pas, ou ne peuvent pas, s'abonner simultanément aux deux outils. Il existe cependant une autre ressource, bien modeste il est vrai, mais bien adaptée au contexte de l'Amérique du Nord francophone: l'Index des revues de la Fédération québécoise des sociétés de généalogie (FQSG) <http://www.federationgenealogie. qc.ca/indexdesrevues $/>^{10}$. Plus jeune que PERSI, cet outil produit depuis 2011 sous la direction de la FQSG, de la SGCF et de la SGQ, et avec la coopération de l'ensemble des sociétés de généalogies du Québec, recense près de 500 titres canadiens, américains et européens et donne accès à des articles datés de 1940 à aujourd'hui ${ }^{11}$.

\section{Des ressources dépassées?}

Même si les périodiques en généalogie offrent une abondance d'information, ils sont souvent négligés par les chercheurs. Pourtant, ils permettent d'aller au-delà des sources traditionnelles (archives notariales et d'état civil, répertoires de registres paroissiaux, etc.). Bref, ils sont fort utiles pour apporter des détails difficiles à trouver et pour fournir une mise en contexte de la vie d'un ancêtre. Il y a dans ces documents une réelle volonté de transmettre un savoir.

Tout comme dans les autres domaines scientifiques, les périodiques en généalogie sous forme imprimée sont confrontés à bien des défis ces dernières années. Avec l'abondance croissante des ressources en ligne pertinentes à l'histoire des familles, les différents acteurs du domaine ont peine à retenir leurs membres. Naturellement, les publications ne peuvent qu'être affectées par la perte de ressources financières qui en résulte: changement de format, réduction du nombre de pages, abandon de la version imprimée, etc. L'avenir de ces documents est-il pour autant sombre? Difficile à dire. Il reste que la masse documentaire produite ces dernières décennies par ces périodiques peut dans certains cas demeurer pertinente aux chercheurs bien après leur publication initiale.

10. Par exemple, une base de données d'avis de décès parus dans les journaux. Voir : Info-Généalogie, $\mathrm{FQSC}$, vol. 24, $\mathrm{n}^{\circ}$ 3, septembre 2012, p. 4.

11. Cette ressource a d'abord été mise à la disposition des membres des sociétés participantes. Depuis l'automne 2012, elle est toutefois offerte en libre accès sur le portail de la FQSG. Il est aussi important de noter les différents niveaux de traitement que l'on trouve dans cette base de données. Si tous les articles des titres québécois et canadiens sont traités, ce n'est pas le cas pour les revues étrangères, où seuls les textes ayant pour sujet le Canada, le Québec ou les Canadiens français sont retenus. Voir : Info-Généalogie, FQSG, vol. 23, $\mathrm{n}^{\circ} 3$, septembre 2011, p. 2.
Sources consultées

Baboyanté, M. 1996. La Bibliothèque de la Ville de Montréal, la collection Gagnon et son fondateur Philéas Gagnon. Cahiers d'histoire du Québec au $x x^{e}$ siècle, 6: 67-82.

Cooke, L.L. 2012. Everything you need to know about... How to find your family history in newspapers, [United States]. Lisa Louise Cooke.

Fédération québécoise des sociétés de généalogie. Revues intéressées à la généalogie québécoise. In Guide des ressources. En ligne : <http://www.federationgenealogie.qc.ca/index. php ?option $=$ com_content $\&$ view $=$ article $\&$ id $=118 \&$ Itemid $=205$ \&lang $=$ fr $>$ [consulté le 20 octobre 2012].

Hinckley, K.W. 2011. Finding Ancestors in Periodicals. What You'll Find in Genealogy Magazines, Ancestry.com. En ligne : $<$ http://www.genealogy.com/34_kathy.html> [consulté le 31 octobre 2012].

Presse généalogique. In GENEAWIKI.COM. En ligne : <http:// fr.geneawiki.com/index.php/Presse_généalogique $>$ [consulté le 31 octobre 2012].

Simpson, J. 2008. Basics of genealogy reference : a librarian's guide. Westport (Conn.), Libraries Unlimited.

Swan, J. 2004. The librarian's guide to genealogical services and research. New York, Neal-Schuman.

Walsh, B.B. 1983. Genealogical Periodicals : A Guide for Research, A revision. Research Guide, ${ }^{\circ}$ 24. En ligne : <http://www. loc.gov/rr/genealogy/bib_guid/genperio.html> [consulté le 31 octobre 2012].

Woods, R.J. et H.B. Hoff. 2011. Looking for Genealogical Journals Online ? In Internet Genealogy, décembre-janvier, 18-21.

Wynkoop, C.H. 2005. Genealogy Periodical Indexes, PERSI, the GPAI and AGBI. En ligne : <http://freepages.genealogy. rootsweb.ancestry.com/ wynkoop/webdocs/persi.htm> [consulté le 31 octobre 2012].

Périodiques cités [URL validés le 5 novembre 2012]

Afro-American Historical and Genealogical Society Journal (1980 ?- ), Afro-Americain Historical and Genealogical Society : <http:// www.aahgs.org/journal.htm>.

American Ancestors (2000-), New England Historic Genealogical Society : <http://www.americanancestors.org/ american-ancestors-magazine/ $>$.

American-Canadian Genealogist (1973- ), American-Canadian Genealogical Society : <http://acgs.org/genealogist/index. html>.

L’Ancêtre (1974- ), Société de généalogie de Québec : <http://www. sgq.qc.ca/revue-ancetre/ancetre-en-ligne.html $>$.

Avotaynu (1985- ), Avotaynu : <http://www.avotaynu.com/>.

Bulletin des recherches historiques (1895-1968), Pierre-Georges Roy: s/o.

The British Colombia Genealogist (1971- ), British Colombia Genealogical Society: <http://www.bcgs.ca/ ?page_id=253>.

Les Cahiers des Dix (1936- ), Société des Dix : <http://www.er.uqam. ca/nobel/sodixi/>.

Les Cahiers (1961- ), Société historique acadienne : s/o.

Le Chaînon (1983-), Réseau du patrimoine franco-ontarien : <http:// www.rpfo.ca/fr/Le-Chainon_26>.

Connections (1978 - ), Quebec Family History Society : <http://www. qfhs.ca/>.

Families (1971- ), Ontario Genealogical Society : <http://www.ogs. on.ca/membership/families.php >.

Family Chronicle (1996-), Moorshead Magazines : <https ://www. familychronicle.com/>.

Family Tree Magazine (1984- ), ABM Publishing Ltd. : <http:// family-tree.co.uk/>.

French Canadian and Acadian Genealogical Review (1968-1981), Centre canadien des recherches généalogiques, Québec : s/o. 
Généalogie Magazine (1982-), Éditions Christian : <http://www. genealogiemagazine.com/>.

Generations (1976- ), Manitoba Genealogical Society : <http://www. mbgenealogy.com/index.php ?page=generations $>$.

Generations (1983-), New Brunswick Genealogical Society : <http:// www.nbgs.ca/publications.html>.

Germaniques, Ahnengalerie (1981- ), Association des familles d'origine germanique du Québec : s/o.

Internet Genealogy (2006- ), Moorshead Magazines : <http://internet-genealogy.com/>.

Mémoires, Société généalogique canadienne-française, Montréal : $<$ http://www.sgcf.com>.

The New England Historical and Genealogical Register (1847-), New England Historic Genealogical Society : <http://www.americanancestors.org/the-register/>.

The Nova Scotia Genealogist (1983-), Genealogical Association of Nova Scotia : <http://www.novascotiaancestors.ca/cpage. php ?pt $=31>$.

NGS Magazine (1974 ?- ), National Genealogical Society : <http:// www.ngsgenealogy.org/cs/ngs_news_magazine $>$.

Links (1996-), Vermont French-Canadian Genealogical Society: <http://www.vt-fcgs.org/journal.html>.

Quartely (1987-2012 ?), French Canadian / Acadian Genealogists of Wisconsin : s/o.

La revue (1982- ), Société historique du Madawaska : $<$ http://www. demelerlespinceaux.ca/societe-historique-du-madawaska $>$.

La revue (1970-), Société historique Nicolas Denys : $<$ http://www. umoncton.ca/umcs-bibliotheque/node/6>.

Revue française de généalogie (1979- ), MartinMedia [France] : $<$ http://www.rfgenealogie.com/>.

Revue d'histoire de l'Amérique française (1947- ), Institut d'histoire de l'Amérique française : <http://www.ihaf.qc.ca >.

Rodziny (1977 ?- ), Polish Genealogical Society of America : <http:// www.pgsa.org/Publications/publications.php>.

Sur l'empremier (1981-), Société historique de la Mer Rouge [Shédiac, Nouveau-Brunswick] : <http://www.lapicasse.ca/la_picasse/ index.cfm $? \mathrm{id}=3460>$.

Swedish American Genealogist (1981-), Swenson Center : <http:// www.augustana.edu/x13918.xml>.

Catalogues de bibliothèques et bases de données [url validés le 5 novembre 2012]

AMICUS, catalogue de Bibliothèque et Archives Canada, <www. amicus.collectionscanada.ca>.

Astrolabe, catalogue des Bibliothèques de la ville de Québec : $<$ http://catalogue.bibliothequesdequebec.qc.ca/>.

Bibliothèque Lisette-Morin, catalogue de la bibliothèque de la Ville de Rimouski : <http://biblio.ville.rimouski.qc.ca/>.

Iris, catalogue de Bibliothèque et Archives nationales du Québec: $<$ www.iris.banq.qc.ca $>$.

ISSN International Center, portail du Centre international de l'ISSN : <www.issn.org >.

Léonard, catalogue des Bibliothèques de la Ville de Longueuil : $<\mathrm{http} / / /$ bibliotheques.longueuil.ca/ $>$.

Nelligan, catalogue des Bibliothèques de la Ville de Montréal : $<\mathrm{http}$ ://nelligan.ville.montreal.qc.ca/>.

Sésame, catalogue des Bibliothèques de la Ville de Laval : <http:// www.biblio.ville.laval.qc.ca/ZonesL/>.

Ulrichsweb.com, répertoire Web de périodiques : $<w w w . u l r i c h s w e b$. serialssolutions.com>. 